\title{
On a class of analytic functions related to Robertson's formula and subordination
}

\section{Adam Lecko $^{1}$ (D) Gangadharan Murugusundaramoorthy ${ }^{2}$. Srikandan Sivasubramanian ${ }^{3}$}

Received: 7 October 2020 / Accepted: 25 November 2020/Published online: 23 February 2021

(C) The Author(s) 2021

\begin{abstract}
In this paper, we define and study a class of analytic functions in the unit disc by modification of the well-known Robertson's analytic formula for starlike functions with respect to a boundary point combined with subordination. An integral representation and growth theorem are proved. Early coefficients and the Fekete-Szegö functional are also estimated.
\end{abstract}

Keywords Univalent function - Starlike function of order $\alpha \cdot$ Starlike function with respect to a boundary point - Coefficient estimates

Mathematics Subject Classification 30C45 $\cdot 30 \mathrm{C} 50 \cdot 30 \mathrm{C} 80$

The work of the third author is supported by a grant from the Science and Engineering Research Board, Government of India under Mathematical Research Impact Centric Support of Department of Science and Technology (DST) (vide ref: MTR/2017/000607). The authors want to thank the reviewer for improving the manuscript.

Adam Lecko

alecko@matman.uwm.edu.pl

Gangadharan Murugusundaramoorthy

gmsmoorthy@yahoo.com

Srikandan Sivasubramanian

sivasaisastha@rediffmail.com

1 Department of Complex Analysis, Faculty of Mathematics and Computer Science, University of Warmia and Mazury in Olsztyn, ul. Słoneczna 54, 10-710 Olsztyn, Poland

2 Department of Mathematics, School of Advanced Sciences, Vellore 632 014, India

3 Department of Mathematics, University College of Engineering Tindivanam, Anna University, Tindivanam 604001, India 


\section{Introduction}

Let $\mathcal{H}$ denote the class of all holomorphic functions in the open unit disc $\mathbb{D}:=\{z \in \mathbb{C}:|z|<1\}$. By $\mathcal{A}$ we denote the subclass of $\mathcal{H}$ of all functions $h$ normalized by $h(0)=0$ and $h^{\prime}(0)=1$, i.e., of the form

$$
h(z)=z+\sum_{n=2}^{\infty} a_{n} z^{n}, \quad z \in \mathbb{D},
$$

and by $\mathcal{S}$ the subclass of $\mathcal{A}$ of univalent functions. We say that a function $f \in \mathcal{H}$ is subordinate to a function $g \in \mathcal{H}$ and write $f \prec g$ if there exists a function $\omega \in \mathcal{H}$ such that $\omega(0)=0, \omega(\mathbb{D}) \subset \mathbb{D}$ and $f(z)=g(\omega(z))$ for every $z \in \mathbb{D}$. In case when $g$ is univalent, then $f \prec g$ if and only if $f(0)=g(0)$ and $f(\mathbb{D}) \subset g(\mathbb{D})$.

Let us denote by $\mathcal{P}$ the class of functions $p \in \mathcal{H}$ normalized by $p(0)=1$ and such that $\mathfrak{R} \mathfrak{p}(\mathfrak{z})>$ for $z \in \mathbb{D}$, which is known as the Carathéodory class. By $\mathcal{P}^{*}(1)$ we denote the subclass of $\mathcal{P}$ of all $\phi$ such that $\phi(0)=1, \phi^{\prime}(0)>0, \phi$ is univalent in $\mathbb{D}$ and $\phi(\mathbb{D})$ is a set symmetric with respect to the real axis and starlike with respect to 1. Thus, every $\phi \in \mathcal{P}^{*}(1)$ can be represented as

$$
\phi(z)=1+\sum_{n=1}^{\infty} B_{n} z^{n}, \quad z \in \mathbb{D},
$$

with $B_{1}>0$.

Given $\phi \in \mathcal{P}^{*}(1)$, let $\mathcal{P}(\phi):=\{p \in \mathcal{P}: p \prec \phi\}$. The class $\mathcal{P}^{*}(1)$ plays a fundamental role in defining suitable classes of analytic functions as was proposed first by Ma and Minda [14]. As an example, given $\phi \in \mathcal{P}^{*}(1)$, let $\mathcal{S}^{*}(\phi)$ denote the class of all $f \in \mathcal{A}$ such that $z f^{\prime}(z) / f(z) \prec \phi(z)$ for $z \in \mathbb{D}$. Such defined classes are called of Ma and Minda type.

The two well-known subclasses of $\mathcal{A}$, are namely the class of starlike and convex functions of order $\alpha(0 \leq \alpha<1)$ introduced by Robertson [18] given, respectively, by

$$
\mathcal{S}^{*}(\alpha):=\left\{h \in \mathcal{A}: \mathfrak{R} \frac{\mathfrak{z}^{\mathfrak{h}^{\prime}(\mathfrak{z})}}{\mathfrak{h}(\mathfrak{z})}>\alpha, \mathfrak{z} \in \mathbb{D}\right\}
$$

and

$$
\mathcal{K}(\alpha):=\left\{h \in \mathcal{A}: \mathfrak{R}\left(+\frac{\mathfrak{z h}^{\prime \prime}(\mathfrak{z})}{\mathfrak{h}^{\prime}(\mathfrak{\jmath})}\right)>\alpha, \mathfrak{z} \in \mathbb{D}\right\} .
$$

It is well known that $\mathcal{S}^{*}(\alpha) \subset \mathcal{S}$ and $\mathcal{K}(\alpha) \subset \mathcal{S}$. By virtue of the well-known Alexander's relation, we see that $h \in \mathcal{K}(\alpha)$ in $\mathbb{D}$ if and only if the function $\mathbb{D} \ni$ $z \mapsto z h^{\prime}(z) \in \mathcal{S}^{*}(\alpha)$ for each $0 \leq \alpha<1$. It is clear that $\mathcal{S}^{*}(\alpha)=\mathcal{S}^{*}(\phi)$ with $\phi(z)=(1+z) /(1-z), z \in \mathbb{D}$.

We say that $h \in \mathcal{H}$ is close-to-convex if and only if there exists a function $\Phi \in \mathcal{K}:=\mathcal{K}(0)$ such that

$$
\mathfrak{R} \frac{\mathfrak{h}^{\prime}(\mathfrak{z})}{\Phi^{\prime}(\mathfrak{z})}>, \quad \mathfrak{z} \in \mathbb{D} .
$$

The class of close-to-convex functions was introduced by Kaplan [7]. 
Even though starlikeness of order $\alpha$ has been explored extensively by many authors over a long period of time, not much was known about the class of univalent functions $g$ in $\mathcal{H}$ that map the disc $\mathbb{D}$ onto domains $\Omega$ which are starlike with respect to a boundary point. This breakthrough concept was introduced by Robertson [19] who defined the subclass $\mathcal{G}^{*}$ of $\mathcal{H}$ of functions $g$ such that $g(0)=1, g(1):=$ $\lim _{r \rightarrow 1^{-}} g(r)=0, g$ maps univalently $\mathbb{D}$ onto a domain starlike with respect to the origin and $\mathfrak{R}\left(\mathrm{e}^{\mathrm{i} \delta} \mathfrak{g}(\mathfrak{z})\right)>$ for some real $\delta$ and all $z \in \mathbb{D}$. Assume that the constant function $g \equiv 1$ also belongs to $\mathcal{G}^{*}$. Robertson conjectured that the class $\mathcal{G}^{*}$ coincides with the class $\mathcal{G}$ of all $g \in \mathcal{H}$ of the form

$$
g(z)=1+\sum_{n=1}^{\infty} g_{n} z^{n}, \quad z \in \mathbb{D}
$$

such that

$$
\mathfrak{R}\left(\frac{\mathfrak{z} \mathfrak{g}^{\prime}(\mathfrak{z})}{\mathfrak{g}(\mathfrak{z})}+\frac{+\mathfrak{z}}{-\mathfrak{z}}\right)>, \quad \mathfrak{z} \in \mathbb{D}
$$

proving that $\mathcal{G} \subset \mathcal{G}^{*}$. Robertson's conjecture was confirmed by Lyzzaik [13] in 1984, who proved that $\mathcal{G}^{*} \subset \mathcal{G}$. In [19], Robertson proved that if $g \in \mathcal{G}$ and $g \neq 1$, then $g$ is close-to-convex and univalent in $\mathbb{D}$. It is worth mentioning that the analytic condition (3) was known much earlier to Styer [22].

Lecko [9] proposed an alternative analytic characterization of starlike functions with respect to a boundary point proving the necessity, while Lecko and Lyzzaik [12] showing the sufficiency confirmed this new analytic characterizations (see also [10], Chapter VII]). Inspired by Robertson's paper, a class of functions based on the concept of spiral-like domains with respect to a boundary point was introduced by Aharanov et al. [2] (see also [11]).

A closely related class to the class $\mathcal{G}$ is the family $\mathcal{G}(M), M>1$, consisting of all $g \in \mathcal{H}$ of the form (2) such that

$$
\mathfrak{R}\left(\frac{\mathfrak{z} \mathfrak{g}^{\prime}(\mathfrak{z})}{\mathfrak{g}(\mathfrak{z})}+\frac{\mathfrak{z} \mathrm{P}^{\prime}(\mathfrak{z} \mathfrak{M})}{\mathrm{P}(\mathfrak{z} \mathfrak{M})}\right)>, \quad \mathfrak{z} \in \mathbb{D},
$$

introduced by Jakubowski [5]. Here

$$
\mathrm{P}(z ; M):=\frac{4 z}{\left(\sqrt{(1-z)^{2}+4 z / M}+1-z\right)^{2}}, \quad z \in \mathbb{D}
$$

denotes the Pick function. The class

$$
\mathcal{G}(1):=\left\{g \in \mathcal{H}: g(0)=1, \mathfrak{R}\left(\frac{\mathfrak{z} \mathfrak{g}^{\prime}(\mathfrak{z})}{\mathfrak{g}(\mathfrak{z})}+\right)>, \mathfrak{z} \in \mathbb{D}\right\}
$$

was considered in [5] also. Todorov [23] associated the class $\mathcal{G}$ with the functional $f(z) /(1-z)$ for $z \in \mathbb{D}$, and obtained a structured formula and coefficient estimates. Obradovič and Owa [16], and Silverman and Silvia [21] introduced independently the classes $\mathcal{G}_{\alpha}$, where $\alpha \in[0,1)$, of all $g \in \mathcal{H}$ of the form (2) such that 


$$
\mathfrak{R}\left(\frac{\mathfrak{z} \mathfrak{g}^{\prime}(\mathfrak{z})}{\mathfrak{g}(\mathfrak{z})}+(-\alpha) \frac{+\mathfrak{z}}{-\mathfrak{z}}\right)>, \quad \mathfrak{z} \in \mathbb{D} .
$$

Silverman and Silvia [21] observed that for each $\alpha \in[0,1)$ the class $\mathcal{G}_{\alpha}$ is a subclass of $\mathcal{G}^{*}$. Clearly, $\mathcal{G}_{1 / 2}=\mathcal{G}$. Abdullah et al. [1] obtained a few properties and some inequalities related to the functional coefficient associated with the class $\mathcal{G}$. In [6], Jakubowski and Włodarczyk defined the class $\mathcal{G}(A, B)$ for $-1<A \leq 1$ and $-A<B \leq 1$, of all $g \in \mathcal{H}$ of the form (2) such that

$$
\mathfrak{R}\left(\frac{\mathfrak{z} \mathfrak{g}^{\prime}(\mathfrak{z})}{\mathfrak{g}(\mathfrak{z})}+\mathfrak{Q}(\mathfrak{z} \mathfrak{A}, \mathfrak{B})\right)>, \quad \mathfrak{z} \in \mathbb{D},
$$

where

$$
Q(z ; A, B):=\frac{1+A z}{1-B z}, \quad z \in \mathbb{D} .
$$

Using Ma and Minda idea, Mohd and Darus [15] introduced the class $\mathcal{S}_{b}^{*}(\phi)$, where $\phi \in \mathcal{P}^{*}(1)$, of all $g \in \mathcal{H}$ of the form (2) such that

$$
\frac{2 z g^{\prime}(z)}{g(z)}+\frac{1+z}{1-z} \prec \phi(z), \quad z \in \mathbb{D} .
$$

The main goal of this paper is to define and study the following class of functions.

Definition 1 Let $\phi \in \mathcal{P}^{*}(1)$ and $-1<A \leq 1,-A<B \leq 1$. By $\mathcal{G}(\phi ; A, B)$ we denote the class of all $g \in \mathcal{H}$ of the form (2) such that

$$
\frac{2 z g^{\prime}(z)}{g(z)}+Q(z ; A, B) \prec \phi(z), \quad z \in \mathbb{D},
$$

where $Q$ is given by (4).

Remark 1 1. Notice that for formula (6) to be well defined, the function

$$
p(z):=\frac{2 z g^{\prime}(z)}{g(z)}+\frac{1+A z}{1-B z}, \quad z \in \mathbb{D}
$$

should be holomorphic in $\mathbb{D}$. We easily that $g$ does not vanish in $\mathbb{D}$. Indeed, suppose that $g\left(z_{0}\right)=0$ for some $z_{0} \in \mathbb{D}$. Since $g(0)=1, z_{0} \neq 0$. Therefore, there exist $r \in\left(0,1-\left|z_{0}\right|\right)$ and $m \in \mathbb{N}$ such that

$$
g(z)=\left(z-z_{0}\right)^{m} h(z), \quad z \in \mathbb{D}\left(z_{0}, r\right):=\left\{z \in \mathbb{C}:\left|z-z_{0}\right|<r\right\},
$$

where $h \in \mathcal{H}$ and $h\left(z_{0}\right) \neq 0$. Since 


$$
\begin{aligned}
\frac{z g^{\prime}(z)}{g(z)} & =\frac{z\left[m\left(z-z_{0}\right)^{m-1} h(z)+\left(z-z_{0}\right)^{m} h^{\prime}(z)\right]}{\left(z-z_{0}\right)^{m} h(z)} \\
& =\frac{m z}{z-z_{0}}+\frac{z h^{\prime}(z)}{h(z)}, \quad z \in \mathbb{D}\left(z_{0}, r\right) \backslash\left\{z_{0}\right\}
\end{aligned}
$$

we see that $p$ has a simple pole at $z_{0}$ which is impossible. Thus $g(z) \neq 0$ for $z \in \mathbb{D}$.

Note that the class $\mathcal{G}(\phi ; 1,1)$ coincides with the class $\mathcal{S}_{b}^{*}(\phi)$ due to Mohd and Darus [15], the class $\mathcal{G}(Q(\cdot ; 1,1) ; 1,1)$ is identical to the class $\mathcal{G}$ and $\mathcal{G}(Q(\cdot ; 1,1) ; 0,0)=\mathcal{G}(1)$. If $B=-A$, then $Q(\cdot ; A,-A) \equiv 1$ and, therefore, again $\mathcal{G}(Q(\cdot ; A,-A) ; 1,1)=G(1)$.

It is worth reminding in this place that the function (4) was used in many papers, where different classes generated by the appropriate Carathéodory functions were considered. It is to be observed that for $B<1$ the function $Q$ maps univalently the disc $\mathbb{D}$ onto a disc lying in the right half-plane. However if $B=1$, then $Q(\mathbb{D} ; A, B)=\{w: \mathfrak{R}(\mathfrak{w})>(-\mathfrak{U}) /\}$.

\section{Representation and growth theorems}

Let $\phi \in \mathcal{P}^{*}(1)$. Since $1 \in \phi(\mathbb{D})$, it follows that $\mathbb{D}(1, a) \subset \phi(\mathbb{D})$ for some $a>0$. Therefore, for each $n \in \mathbb{N}$ we see that $1+a z^{n} \prec \phi(z), z \in \mathbb{D}$. Given $n \in \mathbb{N}$, consider now $g_{n} \in \mathcal{G}(\phi ; A, B)$ defined by

$$
\frac{2 z g_{n}^{\prime}(z)}{g_{n}(z)}+\frac{1+A z}{1-B z}=1+a z^{n}, \quad z \in \mathbb{D},
$$

i.e., for $B \neq 0$,

$$
g_{n}(z)=(1-B z)^{\frac{A+B}{2 B}} \exp \left(\frac{a}{2 n} z^{n}\right), \quad z \in \mathbb{D},
$$

and for $B=0$,

$$
g_{n}(z)=\exp \left(-\frac{1}{2} A z+\frac{a}{2 n} z^{n}\right), \quad z \in \mathbb{D} .
$$

Hence, for $B \neq 0$,

$$
\left|g_{n}(z)\right| \geq(1-|B| r)^{\frac{A+B}{2 B}} \exp \left(-\frac{a}{2 n} r^{n}\right), \quad|z|=r<1 .
$$

Thus, for $B<1, B \neq 0$,

$$
\left|g_{n}(z)\right| \geq(1-|B|)^{\frac{A+B}{2 B}} \exp \left(-\frac{a}{2 n}\right)>0 \quad z \in \mathbb{D} .
$$

Thus, $g_{n}(\mathbb{D})$ is not a set starlike with respect to a boundary point at the origin, so $g_{n} \notin \mathcal{G}^{*}$ and therefore $g_{n} \notin \mathcal{G}$. In the same way, we deduce that $g_{n} \notin \mathcal{G}$ when $B=0$. Thus, we have 
Theorem 1 Let $\phi \in \mathcal{P}^{*}(1)$ and $-1<A \leq 1,-A<B<1$. Then

$$
\mathcal{G}(\phi ; A, B) \not \subset \mathcal{G} \text {. }
$$

Now we prove the representation theorem which indeed offers a useful technique to construct functions in the class $\mathcal{G}(\phi ; A, B)$.

Theorem 2 Let $\phi \in \mathcal{P}^{*}(1)$ and $-1<A \leq 1,-A<B \leq 1$. Then $g \in \mathcal{G}(\phi ; A, B)$ if and only if there exists a function $p \in \mathcal{H}$ such that $p \prec \phi$ and for $z \in \mathbb{D}$,

$$
g(z)= \begin{cases}(1-B z)^{\frac{A+B}{2 B}} \exp \left(\frac{1}{2} \int_{0}^{z} \frac{p(\zeta)-1}{\zeta} d \zeta\right), & B \neq 0 \\ \exp \left(-\frac{A}{2} z+\frac{1}{2} \int_{0}^{z} \frac{p(\zeta)-1}{\zeta} d \zeta\right), & B=0 .\end{cases}
$$

Proof Assume that $g \in \mathcal{G}(\phi ; A, B)$. Consider the function $p$ defined by (7) which is equivalent to

$$
\frac{2 g^{\prime}(z)}{g(z)}+\frac{A+B}{1-B z}=\frac{p(z)-1}{z}, \quad z \in \mathbb{D} .
$$

Clearly, $p \prec \phi$.

When $B \neq 0$, then by integration (9), one can easily get

$$
\log \frac{(g(z))^{2}}{(1-B z)^{\frac{A+B}{B}}}=\int_{0}^{z} \frac{p(\zeta)-1}{\zeta} \mathrm{d} \zeta, \quad z \in \mathbb{D}, \log 1:=0 .
$$

Hence, we obtain

$$
(g(z))^{2}=(1-B z)^{\frac{A+B}{B}} \exp \left(\int_{0}^{z} \frac{p(\zeta)-1}{\zeta} \mathrm{d} \zeta\right), \quad z \in \mathbb{D},
$$

which yields the first formula in (8).

For $B=0$, by virtue of (9), we obtain

$$
\log (g(z))^{2}+A z=\int_{0}^{z} \frac{p(\zeta)-1}{\zeta} \mathrm{d} \zeta, \quad z \in \mathbb{D}, \log 1:=0 .
$$

Hence,

$$
(g(z))^{2}=\exp \left(-A z+\int_{0}^{z} \frac{p(\zeta)-1}{\zeta} d \zeta\right), \quad z \in \mathbb{D},
$$

which yields the second formula in (8).

Assume now that $p \in \mathcal{H}$ is such that $p \prec \phi$ and a function $g$ is defined by (8). Since $\phi(0)=1, p(0)=1$ and, therefore, $g$ is holomorphic in $\mathcal{D}$. As we see, (8) can 
transformed to the formula (7). Hence, we deduce that $g \in \mathcal{G}(\phi ; A, B)$ which completes the proof.

Define $h_{\phi}$ as a holomorphic solution of the differential equation

$$
\frac{z h_{\phi}^{\prime}(z)}{h_{\phi}(z)}=\phi(z), \quad z \in \mathbb{D}, h_{\phi}(0)=0, h_{\phi}^{\prime}(0)=1,
$$

i.e.,

$$
h_{\phi}(z)=z \exp \left(\int_{0}^{z} \frac{\phi(\zeta)-1}{\zeta} d \zeta\right), \quad z \in \mathbb{D} .
$$

Theorem 3 Let $\phi \in \mathcal{P}^{*}(1)$ and $-1<A \leq 1,-A<B \leq 1$ and let $0<r<1$. If $g \in$ $\mathcal{G}(\phi ; A, B)$, then for $B \neq 0$,

$$
\sqrt{\frac{-h_{\phi}(-r)}{r}}(1-|B| r)^{\frac{A+B}{2 B}} \leq|g(z)| \leq \sqrt{\frac{h_{\phi}(-r)}{r}}(1+|B| r)^{\frac{A+B}{B}}, \quad|z|=r,
$$

and for $B=0$,

$$
\sqrt{\frac{-h_{\phi}(-r)}{r}} \exp \left(-\frac{1}{2}|A| r\right) \leq|g(z)| \leq \sqrt{\frac{h_{\phi}(-r)}{r}} \exp \left(\frac{1}{2}|A| r\right), \quad|z|=r
$$

Proof Consider $B \neq 0$. Define

$$
h(z):=\frac{z(g(z))^{2}}{(1-B z)^{\frac{A+B}{B}}}, \quad z \in \mathbb{D} .
$$

By Remark 1, the function $g$ is non-vanishing in $\mathbb{D}$. Therefore, $h$ is holomorphic in $\mathbb{D}$. A simple calculation shows that

$$
\frac{z h^{\prime}(z)}{h(z)}=\frac{2 z g^{\prime}(z)}{g(z)}+\frac{1+A z}{1-B z}, \quad z \in \mathbb{D} .
$$

One can see from the above relation that $g \in \mathcal{G}(\phi ; A, B)$ if and only if $h \in \mathcal{S}^{*}(\phi)$. Using the result of Ma and Minda ([14], Corollary 1') we deduce that

$$
-h_{\phi}(-r) \leq|h(z)| \leq h_{\phi}(r), \quad|z|=r,
$$

i.e., by (13,

$$
-h_{\phi}(-r) \leq\left|\frac{z(g(z))^{2}}{(1-B z)^{\frac{A+B}{B}}}\right| \leq h_{\phi}(r), \quad|z|=r,
$$

which yields (11).

Consider $B=0$. Define 


$$
h(z):=z \exp (A z)(g(z))^{2}, \quad z \in \mathbb{D} .
$$

Clearly, $h$ is holomorphic in $\mathbb{D}$ and

$$
\frac{z h^{\prime}(z)}{h(z)}=\frac{2 z g^{\prime}(z)}{g(z)}+1+A z, \quad z \in \mathbb{D} .
$$

Arguing as above, we see that the condition (15) holds and consequently by (16),

$$
-h_{\phi}(-r) \leq\left|z \exp (A z)(g(z))^{2}\right| \leq h_{\phi}(r), \quad|z|=r,
$$

which yields (12).

Remark 2 As Ma an Minda noted ([14], p. 161) a function $r \longmapsto-h_{\phi}(-r)$ for $r \in$ $(0,1)$, is increasing and bounded above by 1 .

Theorem 4 Let $\phi \in \mathcal{P}^{*}(1)$ and $-1<A \leq 1,-A<B<1$ and let $0<r<1$. If $g \in$ $\mathcal{G}(\phi ; A, B)$, then for $B \neq 0$,

$$
\left|\arg \frac{g\left(z_{0}\right)}{\left(1-B z_{0}\right)^{\frac{A+B}{2 B}}}\right| \leq \frac{1}{2} \max _{|z|=r} \arg \frac{h_{\phi}(z)}{z}, \quad\left|z_{0}\right|=r,
$$

and for $B=0$,

$$
\left|\arg \left(g\left(z_{0}\right) \exp \left(\frac{A z_{0}}{2}\right)\right)\right| \leq \frac{1}{2} \max _{|z|=r} \arg \frac{h_{\phi}(z)}{z}, \quad\left|z_{0}\right|=r,
$$

where $\arg 1:=0$.

Proof Define a function $h$ by (13) in case when $B \neq 0$, and by (16) in case when $B=0$. Clearly, in both cases, $h \in \mathcal{S}^{*}(\phi)$. Thus, in view of a result due to Ma and Minda ([14], Corollary 3'), the following inequality holds

$$
\left|\arg \frac{h\left(z_{0}\right)}{z_{0}}\right| \leq \max _{|z|=r} \arg \frac{h_{\phi}(z)}{z}, \quad\left|z_{0}\right|=r,
$$

which by substituting (13) and (16) yields (17) and (18), respectively.

\section{Necessary and sufficient condition for the class $\mathcal{G}(\phi ; A, B)$}

We state the following result due to Ruscheweyh ([20], Theorem 1) for proving our main theorem in this section. Recall that $h_{\phi}$ which appears below is defined by (10).

Lemma 1 Let $\phi \in \mathcal{P}^{*}(1)$ be a convex function and $h \in \mathcal{A}$. Then $h \in \mathcal{S}^{*}(\phi)$ if and only if for all $|s| \leq 1$ and $|t| \leq 1$,

$$
\frac{h(s z)}{h(t z)} \prec \frac{h_{\phi}(s z)}{h_{\phi}(t z)}, \quad z \in \mathbb{D} .
$$


Theorem 5 Let $\phi \in \mathcal{P}^{*}(1)$ be a convex function and $-1<A \leq 1,-A<B \leq 1$. Then $g \in \mathcal{G}(\phi ; A, B)$ if and only if for all $|s| \leq 1$ and $|t| \leq 1$,

$$
\frac{s}{t}\left(\frac{1-B t z}{1-B s z}\right)^{\frac{A+B}{B}}\left(\frac{g(s z)}{g(t z)}\right)^{2} \prec \frac{h_{\phi}(s z)}{h_{\phi}(t z)}, \quad z \in \mathbb{D}, B \neq 0,
$$

and

$$
\frac{s}{t} \exp (A(s-t) z)\left(\frac{g(s z)}{g(t z)}\right)^{2} \prec \frac{h_{\phi}(s z)}{h_{\phi}(t z)}, \quad z \in \mathbb{D}, B=0 .
$$

Proof Consider the function $h$ defined by (13) in case when $B \neq 0$, and by (16) in case when $B=0$. As we know, in both cases $h \in \mathcal{S}^{*}(\phi)$. Thus, in case $B \neq 0$ by substituting (13) into (19), we get at once (20). Similarly, when $B=0$ by substituting (16) into (19), we obtain (21).

In the previous theorem, we assumed that the function $\phi \in \mathcal{P}^{*}(1)$ is convex univalent. Now, we drop the assumption of convexity on $\phi$ and we return to the whole class $\mathcal{P}^{*}(1)$.

Theorem 6 Let $\phi \in \mathcal{P}^{*}(1)$ and $-1<A \leq 1,-A<B \leq 1$. If $g \in \mathcal{G}(\phi ; A, B)$, then

$$
\frac{(g(z))^{2}}{(1-z)^{\frac{A+B}{B}}} \prec \frac{h_{\phi}(z)}{z}, \quad z \in \mathbb{D}, B \neq 0,
$$

and

$$
\exp (A z)(g(z))^{2} \prec \frac{h_{\phi}(z)}{z}, \quad z \in \mathbb{D}, B=0
$$

Proof Consider the function $h$ defined by (13) in case when $B \neq 0$, and by (16) in case when $B=0$. In view of (14), we have

$$
\frac{z h^{\prime}(z)}{h(z)} \prec \frac{z h_{\phi}^{\prime}(z)}{h_{\phi}(z)}=\phi(z), \quad z \in \mathbb{D} .
$$

Since $h \in \mathcal{S}^{*}(\phi)$, from Theorem 1' of [14] it follows that

$$
\frac{h(z)}{z} \prec \frac{h_{\phi}(z)}{z}, \quad z \in \mathbb{D},
$$

i.e., (22) and (23) by substituting (13) and (16), respectively. 


\section{Initial coefficient bounds for the class $\mathcal{G}(\phi, A, B)$}

In this section, making use of the following Lemmas, we obtain a few coefficient estimates for $g \in \mathcal{G}(\phi ; A, B)$. Let $\mathcal{B}:=\{\omega \in \mathcal{H}:|\omega(z)|<1, z \in \mathbb{D}\}$ and $\mathcal{B}_{0}$ be the subclass of $\mathcal{B}$ of all $\omega$ such that $\omega(0)=0$. Elements of $\mathcal{B}_{0}$ are known as Schwarz functions.

To prove the main theorem of this section, we will use the next two lemmas.

Lemma 2 ([8]) If $\omega \in \mathcal{B}_{0}$ is of the form

$$
\omega(z)=\sum_{n=1}^{\infty} w_{n} z^{n}, \quad z \in \mathbb{D},
$$

then for $v \in \mathbb{C}$,

$$
\left|w_{2}-v w_{1}^{2}\right| \leq \max \{1,|v|\}
$$

The following lemma was shown by Prokhorov and Szynal [17].

Lemma 3 ([17]) If $\omega \in \mathcal{B}$, then for any real numbers $q_{1}$ and $q_{2}$, the following sharp estimate holds:

$$
\left|w_{3}+q_{1} w_{1} w_{2}+q_{2} w_{1}^{3}\right| \leq H\left(q_{1}, q_{2}\right)
$$

where

$$
H\left(q_{1}, q_{2}\right):= \begin{cases}1 & \text { for }\left(q_{1}, q_{2}\right) \in D_{1} \cup D_{2}, \\ \left|q_{2}\right| & \text { for }\left(q_{1}, q_{2}\right) \in \cup_{k=3}^{7} D_{k}, \\ \frac{2}{3}\left(\left|q_{1}\right|+1\right)\left(\frac{\left|q_{1}\right|+1}{3\left(\left|q_{1}\right|+1+q_{2}\right)}\right)^{\frac{1}{2}} & \text { for }\left(q_{1}, q_{2}\right) \in D_{8} \cup D_{9}, \\ \frac{q_{2}}{3}\left(\frac{q_{1}^{2}-4}{q_{1}^{2}-4 q_{2}}\right)\left(\frac{q_{1}^{2}-4}{3\left(q_{2}-1\right)}\right)^{\frac{1}{2}} & \text { for }\left(q_{1}, q_{2}\right) \in D_{10} \cup D_{11} \backslash\{ \pm 2,1\}, \\ \frac{2}{3}\left(\left|q_{1}\right|-1\right)\left(\frac{\left|q_{1}\right|-1}{3\left(\left|q_{1}\right|-1-q_{2}\right)}\right)^{\frac{1}{2}} & \text { for }\left(q_{1}, q_{2}\right) \in D_{12},\end{cases}
$$

and the sets $D_{k}, k=1,2, \ldots, 12$, are defined in [17].

Now we present some upper bounds for early coefficients and for the FeketeSzegö functional in the class $\mathcal{G}(\phi ; A, B)$.

Theorem 7 Let $\phi \in \mathcal{P}^{*}(1)$ be of the form (1) and $-1<A \leq 1,-A<B \leq 1$. If $g \in$ $\mathcal{G}(\phi ; A, B)$ is of the form (2), then

$$
\begin{gathered}
\left|2 d_{1}+A+B\right| \leq B_{1}, \\
\left|d_{1}\right| \leq \frac{1}{2}\left(B_{1}+A+B\right),
\end{gathered}
$$




$$
\begin{gathered}
\left|4 d_{2}-2 d_{1}^{2}+(A+B) B\right| \leq \max \left\{B_{1}, B_{2}\right\}, \\
\left|d_{2}\right| \leq \frac{1}{8}\left[\left|A^{2}-B^{2}\right|+2(A+B) B_{1}+\max \left\{2,\left|2 B_{2}+B_{1}^{2}\right|\right\}\right], \\
\left|6 d_{3}-6 d_{1} d_{2}+2 d_{1}^{3}+(A+B) B^{2}\right| \leq B_{1} H\left(\frac{2 B_{2}}{B_{1}}, \frac{B_{3}}{B_{1}}\right)
\end{gathered}
$$

and

$$
\begin{aligned}
\left|d_{3}\right| \leq & \frac{1}{6}\left[B_{1} H\left(2 \frac{B_{2}}{B_{1}}+\frac{3}{4} B_{1}, \frac{B_{3}}{B_{1}}+\frac{3}{4} B_{2}+\frac{1}{8} B_{1}^{2}\right)\right. \\
& +\frac{3}{8}(A+B) \max \left\{2,\left|2 B_{2}+B_{1}^{2}\right|\right\} \\
& \left.+\frac{3}{8}\left|A^{2}-B^{2}\right| B_{1}+\frac{1}{2}(A+B)\left|A^{2}-4 A B+3 B^{2}\right|\right]
\end{aligned}
$$

and for $\delta \in \mathbb{R}$,

$$
\begin{aligned}
\left|d_{2}-\delta d_{1}^{2}\right| \leq & \frac{1}{4}\left[B_{1} \max \left\{1,\left|\frac{B_{2}}{B_{1}}+\left(\frac{1}{2}-\delta\right) B_{1}\right|\right\}+(A+B)|1-2 \delta| B_{1}\right. \\
& \left.+\frac{1}{2}(A+B)|A-B-2 \delta(A+B)|\right]
\end{aligned}
$$

Proof By (5), there exists $w \in \mathcal{B}_{0}$ of the form (24) such that

$$
\frac{2 z g^{\prime}(z)}{g(z)}+\frac{1+A z}{1-B z}=\phi(w(z)), \quad z \in \mathbb{D} .
$$

In view of (2), we obtain

$$
\begin{aligned}
\frac{2 z g^{\prime}(z)}{g(z)}+ & \frac{1+A z}{1-B z}=1+\left(2 d_{1}+A+B\right) z+\left[4 d_{2}-2 d_{1}^{2}+(A+B) B\right] z^{2} \\
& +\left[6 d_{3}-6 d_{1} d_{2}+2 d_{1}^{3}+(A+B) B^{2}\right]+\cdots, \quad z \in \mathbb{D} .
\end{aligned}
$$

By (1) and (24) for $z \in \mathbb{D}$, we have

$$
\phi(w(z))=1+B_{1} w_{1} z+\left(B_{1} w_{2}+B_{2} w_{1}^{2}\right) z^{2}+\left(B_{1} w_{3}+2 B_{2} w_{1} w_{2}+B_{3} w_{1}^{3}\right) z^{3}+\cdots
$$

Using now (35), (36) and (34) by comparing corresponding coefficients, we get 


$$
\begin{aligned}
& 2 d_{1}+A+B=B_{1} w_{1}, \\
& 4 d_{2}-2 d_{1}^{2}+(A+B) B=B_{1} w_{2}+B_{2} w_{1}^{2}, \\
& 6 d_{3}-6 d_{1} d_{2}+2 d_{1}^{3}+(A+B) B^{2}=B_{1} w_{3}+2 B_{2} w_{1} w_{2}+B_{3} w_{1}^{3} .
\end{aligned}
$$

Recall that $B_{1}=\phi^{\prime}(0)>0$. Since

$$
\left|w_{1}\right| \leq 1
$$

(e.g., [4], Vol. I, p. 85]) from the first equation in (37) it follows (27) and hence (28).

The second equation in (37) together with (25) yields

$$
\begin{aligned}
\left|4 d_{2}-2 d_{1}^{2}+(A+B) B\right| & =\left|B_{1} w_{2}+B_{2} w_{1}^{2}\right| \\
& =B_{1}\left|w_{2}+\frac{B_{2}}{B_{1}} w_{1}\right| \leq B_{1} \max \left\{1, \frac{B_{2}}{B_{1}}\right\}=\max \left\{B_{1}, B_{2}\right\},
\end{aligned}
$$

i.e., the inequality (29).

Substituting the first formula in (37) for $d_{1}$ into the second formula in (37), we obtain

$$
4 d_{2}=B_{1}\left[w_{2}+\left(\frac{B_{2}}{B_{1}}+\frac{1}{2} B_{1}\right) w_{1}^{2}\right]-(A+B) B_{1} w_{1}+\frac{1}{2}\left(A^{2}-B^{2}\right) .
$$

Hence, using (25) and (38), we get

$$
4\left|d_{2}\right| \leq B_{1} \max \left\{1,\left|\frac{B_{2}}{B_{1}}+\frac{1}{2} B_{1}\right|\right\}+(A+B) B_{1}+\frac{1}{2}\left|A^{2}-B^{2}\right|,
$$

which yields (30).

The third equation in (37) by applying (26) yield

$$
\begin{aligned}
\left|6 d_{3}-6 d_{1} d_{2}+2 d_{1}^{3}+(A+B) B^{2}\right| & =\left|B_{1} w_{3}+2 B_{2} w_{1} w_{2}+B_{3} w_{1}^{3}\right| \\
& =B_{1}\left|w_{3}+2 \frac{B_{2}}{B_{1}} w_{1} w_{2}+\frac{B_{3}}{B_{1}} w_{1}^{3}\right| \leq B_{1} H\left(\frac{2 B_{2}}{B_{1}}, \frac{B_{3}}{B_{1}}\right),
\end{aligned}
$$

i.e., the inequality (31).

Substituting formulas for $d_{1}$ and $d_{2}$ as in (39) into the third formula in (37), we obtain

$$
\begin{aligned}
6 d_{3}= & B_{1} w_{3}+\left(2 B_{2}+\frac{3}{4} B_{1}^{2}\right) w_{1} w_{2}+\left(B_{3}+\frac{3}{4} B_{1} B_{2}+\frac{1}{8} B_{1}^{3}\right) w_{1}^{3} \\
& -\frac{3}{4}(A+B) B_{1} w_{2}-\frac{3}{8}(A+B)\left(2 B_{2}+B_{1}^{2}\right) w_{1}^{2}+\frac{3}{8}\left(A^{2}-B^{2}\right) B_{1} w_{1} \\
& -\frac{1}{2}(A+B)\left(A^{2}-4 A B+3 B^{2}\right) .
\end{aligned}
$$

Now applying (25), (26) and (38), we get 


$$
\begin{aligned}
6\left|d_{3}\right| \leq & B_{1}\left|w_{3}+\left(2 \frac{B_{2}}{B_{1}}+\frac{3}{4} B_{1}\right) w_{1} w_{2}+\left(\frac{B_{3}}{B_{1}}+\frac{3}{4} B_{2}+\frac{1}{8} B_{1}^{2}\right) w_{1}^{3}\right| \\
& +\frac{3}{4}(A+B) B_{1}\left|w_{2}+\left(\frac{B_{2}}{B_{1}}+\frac{1}{2} B_{1}\right) w_{1}^{2}\right|+\frac{3}{8}\left|A^{2}-B^{2}\right| B_{1}\left|w_{1}\right| \\
& +\frac{1}{2}(A+B)\left|A^{2}-4 A B+3 B^{2}\right| \\
\leq & B_{1} H\left(2 \frac{B_{2}}{B_{1}}+\frac{3}{4} B_{1}, \frac{B_{3}}{B_{1}}+\frac{3}{4} B_{2}+\frac{1}{8} B_{1}^{2}\right) \\
& +\frac{3}{4}(A+B) \max \left\{1,\left|B_{2}+\frac{1}{2} B_{1}^{2}\right|\right\} \\
& +\frac{3}{8}\left|A^{2}-B^{2}\right| B_{1}+\frac{1}{2}(A+B)\left|A^{2}-4 A B+3 B^{2}\right|,
\end{aligned}
$$

which yields (32).

Using (39), the formula for $d_{1}$ by applying the inequalities (25) and (38), for $\delta \in \mathbb{R}$, we get

$$
\begin{aligned}
\left|d_{2}-\delta d_{1}^{2}\right| \leq & \frac{1}{4}\left[B_{1}\left|w_{2}+\left(\frac{B_{2}}{B_{1}}+\left(\frac{1}{2}-\delta\right) B_{1}\right) w_{1}^{2}\right|\right. \\
& \left.+(A+B)|1-2 \delta| B_{1}+\frac{1}{2}(A+B)|A-B-2 \delta(A+B)|\right] \\
\leq & \frac{1}{4}\left[B_{1} \max \left\{1,\left|\frac{B_{2}}{B_{1}}+\left(\frac{1}{2}-\delta\right) B_{1}\right|\right\}+(A+B)|1-2 \delta| B_{1}\right. \\
& \left.+\frac{1}{2}(A+B)|A-B-2 \delta(A+B)|\right]
\end{aligned}
$$

i.e., the inequality (33).

In case when $v$ is a real number result of Lemma 2 can be improved in the following way.

Lemma 4 ([3]) If $\omega \in \mathcal{B}_{0}$ is of the form (24), then

$$
\left|w_{2}-v w_{1}^{2}\right| \leq\left\{\begin{array}{cc}
-v, & v \leq-1 \\
1, & -1 \leq v \leq 1 \\
v, & v \geq 1
\end{array}\right.
$$

For $v<-1$ or $v>1$, equality holds if and only if $\omega(z)=z, z \in \mathbb{D}$, or one of its rotations. For $-1<v<1$, equality holds if and only if $\omega(z)=z^{2}, z \in \mathbb{D}$, or one of its rotations. For $v=-1$, equality holds if and only if $\omega(z)=z(\lambda+z) /(1+$ $\lambda z), 0 \leq \lambda \leq 1, z \in \mathbb{D}$, or one of its rotations, while for $v=1$, equality holds if and only if $\omega(z)=-z(\lambda+z) /(1+\lambda z), 0 \leq \lambda \leq 1, z \in \mathbb{D}$, or one of its rotations. 
Taking into account the above lemma, we can improve the results of Theorem 7 . From (39), we have

$$
4\left|d_{2}\right| \leq \begin{cases}B_{2}+\frac{1}{2} B_{1}^{2}+\gamma(A, B), & \frac{B_{2}}{B_{1}}+\frac{1}{2} B_{1} \geq 1, \\ \gamma(A, B), & -1 \leq \frac{B_{2}}{B_{1}}+\frac{1}{2} B_{1} \leq 1, \\ -B_{2}-\frac{1}{2} B_{1}^{2}+\gamma(A, B), & \frac{B_{2}}{B_{1}}+\frac{1}{2} B_{1} \leq-1,\end{cases}
$$

where

$$
\gamma(A, B):=(A+B) B_{1}+\frac{1}{2}\left|A^{2}-B^{2}\right| .
$$

Analogously, by applying (40) for $\delta \in \mathbb{R}$, we obtain

$$
\left|d_{2}-\delta d_{1}^{2}\right| \leq \begin{cases}\frac{B_{1}}{4}\left(\frac{B_{2}}{B_{1}}+\frac{B_{1}}{2}(1-2 \delta)\right)+\Psi(A, B, \delta), & \delta \leq \sigma_{1} \\ \frac{B_{1}}{4}+\Psi(A, B, \delta), & \sigma_{1} \leq \delta \leq \sigma_{2} \\ \frac{B_{1}}{4}\left((2 \delta-1) \frac{B_{1}}{2}-\frac{B_{2}}{B_{1}}\right)+\Psi(A, B, \delta), & \delta \geq \sigma_{2},\end{cases}
$$

where

$$
\sigma_{1}:=\frac{1}{B_{1}}\left(\frac{B_{2}}{B_{1}}-1\right)+\frac{1}{2}, \quad \sigma_{2}:=\frac{1}{B_{1}}\left(\frac{B_{2}}{B_{1}}+1\right)+\frac{1}{2},
$$

and

$$
\Psi(A, B, \delta):=|A(1-2 \delta)-B(1+2 \delta)| \frac{(A+B)}{8}+|1-2 \delta| \frac{(A+B) B_{1}}{4} .
$$

Since

$$
6 d_{3}=B_{1}\left(w_{3}+\frac{B_{2}}{B_{1}} w_{1} w_{2}+\frac{B_{3}}{B_{1}} w_{1}^{3}\right)+6 d_{1}\left(d_{2}-\frac{d_{1}^{2}}{3}\right)-(A+B) B^{2},
$$

using (40) and (41) with $\delta=1 / 3$, we get

$$
\begin{aligned}
\left|d_{3}\right| \leq & \frac{B_{1}}{6} H\left(\frac{B_{2}}{B_{1}}, \frac{B_{3}}{B_{1}}\right)+\left(\frac{|A-5 B|}{3}+\frac{(A+B) B_{1}}{6}\right)+\frac{1}{6}(A+B) B^{2} \\
& +B_{1}\left(\frac{\left(1+B_{1}\right)(A+B)}{8}\right) \max \left\{1,\left|\frac{B_{2}}{B_{1}}+\frac{B_{1}}{6}\right|\right\} .
\end{aligned}
$$

Remark 3 For the choice of $\phi(z)=(1+z) /(1-z), z \in \mathbb{D}$, with $A=1$ and $B=1$, inequalities $(28,(29)$ and (31) reduce to the results of Abdullah et al. [1]. However, the technique adopted in this paper is different. 


\section{Compliance with ethical standards}

Conflict of interest The authors declare that they do not have conflict of interests.

Ethical standard This research complies with ethical standards

Open Access This article is licensed under a Creative Commons Attribution 4.0 International License, which permits use, sharing, adaptation, distribution and reproduction in any medium or format, as long as you give appropriate credit to the original author(s) and the source, provide a link to the Creative Commons licence, and indicate if changes were made. The images or other third party material in this article are included in the article's Creative Commons licence, unless indicated otherwise in a credit line to the material. If material is not included in the article's Creative Commons licence and your intended use is not permitted by statutory regulation or exceeds the permitted use, you will need to obtain permission directly from the copyright holder. To view a copy of this licence, visit http:// creativecommons.org/licenses/by/4.0/.

\section{References}

1. Abdullah, A.S., Ali, R.M., Singh, V.: On functions starlike with respect to a boundary point. Ann. Univ. Mariae Curie-Skłodowska Sect. A 50, 7-15 (1996)

2. Aharonov, D., Elin, M., Shoikhet, D.: Spiral-like functions with respect to a boundary point. J. Math. Anal. Appl. 280(1), 17-29 (2003)

3. Ali, R.M., Ravichandran, V., Seenivasagan, N.: Coefficient bounds for $p$-valent functions. Appl. Math. Comput. 187(1), 35-46 (2007)

4. Goodman, A.W.: Univalent Functions. Mariner, Tampa (1983)

5. Jakubowski, Z.J.: On properties of the Pick function and some applications of them. Acta Universitatis Purkynianae 42, 51-62 (1999)

6. Jakubowski, Z.J., Włodarczyk, A.: On some classes of functions of Robertson type. Ann. Univ. Mariae Curie-Skłodowska, Sectio A Mathematica 59, 27-42 (2005)

7. Kaplan, W.: Close-to-convex schlicht functions. Michigan Math. J. 1, 169-185 (1952)

8. Keogh, F.R., Merkes, E.P.: A coefficient inequality for certain classes of analytic functions. Proc. Am. Math. Soc. 20, 8-12 (1969)

9. Lecko, A.: On the class of functions starlike with respect to a boundary point. J. Math. Anal. Appl. 261(2), 649-664 (2001)

10. Lecko, A.: Some Methods in the Theory of Univalent Functions. Oficyna Wdawnicza Politechniki Rzeszowskiej, Rzeszów (2005)

11. Lecko, A.: $\delta$-spirallike functions with respect to a boundary point. Rocky Mt. J. Math. 38(3), 979-992 (2008)

12. Lecko, A., Lyzzaik, A.: A note on univalent functions starlike with respect to a boundary point. J. Math. Anal. Appl. 282(2), 846-851 (2003)

13. Lyzzaik, A.: On a conjecture of M. S. Robertson. Proc. Am. Math. Soc. 91(1), 108-110 (1984)

14. Ma, W. C., Minda, D.: A unified treatment of some special classes of unvalent functions. In: Proceedings of the Conference on Complex Analysis (Tianjin; June 19-23, 1992) (Zhong Li, Fuyao Ren, Lo Yang and Shunyan Zhang, Editors), Conference Proceedings and Lecture Notes in Analysis, Vol. I, International Press, Cambridge, Massachusetts, 1994, pp. 157-169

15. Mohd, M.H., Darus, M.: Starlike function with respect to a boundary point defined by subordination. Adv. Math. Sci. J. 1(1), 15-21 (2012)

16. Obradovič, M., Owa, S.: On some classes of close-to-convex functions and its applications. Bull. Inst. Math. Acad. Sinica 16(2), 123-133 (2012)

17. Prokhorov, D.V., Szynal, J.: Inverse coefficients for $(\alpha, \beta)$-convex functions. Ann. Univ. Mariae Curie-Skłodowska Sect. A 35(1981), 125-143 (1984)

18. Robertson, M.S.: On the theory of univalent functions. Ann. Math. 37(2), 374-408 (1936)

19. Robertson, M.S.: Univalent functions starlike with respect to a boundary point. J. Math. Anal. Appl. 81(2), 327-345 (1981)

20. Ruscheweyh, S.: A subordination theorem for $\Phi$-like functions. J. Lond. Math. Soc. 13, 275-280 (1976) 
21. Silverman, H., Silvia, E.M.: Subclasses of univalent functions starlike with respect to a boundary point. Houston J. Math. 16(2), 289-299 (1990)

22. Styer, D.: On weakly starlike multivalent functions. J. Anal. Math. 26, 217-233 (1973)

23. Todorov, P.G.: On the univalent functions starlike with respect to a boundary point. Proc. Am. Math. Soc. 97(4), 602-604 (1986)

Publisher's Note Springer Nature remains neutral with regard to jurisdictional claims in published maps and institutional affiliations. 